\title{
Analysis and simulation of fully ankle actuated planar bipedal robots
}

\author{
Michel Franken, Gijs van Oort and Stefano Stramigioli \\ Control Engineering, EE-Math-CS, University of Twente \\ P.O. Box 217, 7500 AE, Enschede, The Netherlands \\ m.c.j.franken@utwente.nl \\ g.vanoortautwente.nl \\ s.stramigioli@utwente.nl
}

\begin{abstract}
This paper deals with the analysis of planar bipedal robots, based on passive dynamic walkers, which are actuated only by actuation of the ankle joints. An overview of the major design characteristics of such robots and their influence on the feasibility of a stable limit cycle is presented. It is shown that robots which are fully powered by ankle actuation require a mass ratio of at least 10:1 between the upper and lower limb to obtain sufficient ground clearance during the swing phase at a wide range of walking speeds. The effect and necessity of the offset in the footshapes of many passive dynamic walkers is shown and the influence of the moment of push off on the required energy injection is treated. The results of the analysis are supported by simulations with a dynamic model of such a robot. The simulated model exhibits a very natural looking gait and walks with a wide range of velocities at low mechanical cost of transport. Simulation results are provided which confirm that pushing off before the swing leg collides with the floor is energetically more efficient than pushing off after the impact as also known from previous literature.
\end{abstract}

\section{INTRODUCTION}

At the Control Engineering (CE) group of the University of Twente research is being conducted in the field of bipedal robots inspired by passive dynamic walkers. Passive dynamic walkers are unactuated walking frames that exhibit a stable and human like gait walking down a slight slope [14]. These robots require only the energy supplied by the reduction in potential energy due to the declining slope and gravity to sustain a stable limit cycle. These walkers rely on the dynamics of the structure for the motion itself and the energy generated by walking down a slope to compensate the loss of energy occuring at each impact of the swing leg with the ground (heel strike).

For a stable limit cycle it is clearly not necessary that the configuration of the robot is stable at all times. This is demonstrated by human walking, which basically consists of a stable series of unstable falling motions [11]. This stable series of falling motions is also found in the limit cycles of passive dynamic walkers

Powered passive dynamic walkers are walking frames that are capable of walking on level ground and use one or more actuators instead of gravity to compensate for the energy lost at heel strike. These robots still rely on the natural dynamics of the frame for walking and only add enough energy to the system to sustain a stable limit cycle. The resulting robots are therefore highly energy efficient compared to traditional walking robots [5]. Some examples are presented in [4][5][6][17].

The current robot, Dribbel, at the CE group [6] uses a hip actuator to inject energy into the sytem by accelerating the new swing leg forward after heel strike of the previous swing leg. Energy however can also be added to the system by pushing off from the ground with the foot of the stance leg. Several studies [13][12] showed that adding energy to the system by means of a push off before impact should be more energetically efficient than other forms of actuation. It would therefore be desirable if the current robot could be extended with actuated ankles to test the energy efficiency and robustness of complex hybrid hip and ankle actuation schemes. This study was performed to provide more insights into the necessary design characteristics of a robot only actuated by ankle actuation.

An analysis of three important elements of ankle actuation is presented in section II. The simulation model of a fully ankle actuated planar bipedal robot is discussed in section III. In section IV the results of the simulations are presented and the paper finishes with conclusions about the presented work and a discussion of future work in sections V and VI.

\section{ANALYSIS}

In this section the functions of the push off will be discussed first and then three important characteristics related to the energy efficiency, the natural dynamics of the system and the robustness of the limit cycle. At the end of the section a comparison will be made with human dynamics.

\section{A. Push off}

As was mentioned the limit cycle of robots based on passive dynamics consists of a series of falling motions. When the swing leg hits the ground the center of mass (c.o.m.) of the robot is redirected from a downward rotation around the trailing leg to an upward rotation around the new stance leg.

The collision with the ground of the swing leg results in negative work, opposite to the direction of movement, on the c.o.m. so that the velocity after impact will have decreased.

A minimum amount of kinetic energy after heel strike is necessary to complete the upward rotation. Since at each heel strike energy is dissipated by the system, reinjection of that energy into the system is necessary, because otherwise the energy of the system will decrease to the point where it is insufficient to sustain a stable limit cycle.

Pushing off with the foot of the stance leg against the ground results in a forward acceleration of the c.o.m. Positive work, in the direction of movement, is therefore performed on the c.o.m. which injects the required energy into the system. 
Another function of the push off is the addition of potential energy to the new swing leg. Actuation of the ankle causes the foot to rotate and the swing leg to rise. This additional potential energy is released when the foot is retracted and is transformed into kinetic energy during the swing phase. In robots in which the energy injection is performed only by ankle actuation the swing phase is completely passive.

\section{B. Moment of push-off}

The collision of the swing leg with the ground results in negative work being performed on the c.o.m. and therefore in a reduction of kinetic energy. The amount of energy loss depends both on the velocity and the angle at which the swing leg hits the ground.

In [13] and [12] the energetic consequences of the moment of push off are analyzed for the simplest walker model with an impulsive push along the stance leg. It is shown that for that model the impact loss is four times smaller with an impulsive push along the stance leg just before heel strike (modelled as instantaneous and perfectly inelastic). An impulsive push along the stance leg redirects the velocity of the swing leg from a downward direction to a direction more parallel with the ground. Figure 1 shows three situations for which the required energy that needs to injected, $W^{+}$is analyzed assuming that both the push off and heel strike are instantaneous impulses, based on [1]. A stable limit cycle requires that the energy injected by the push off equals the energy which is lost due to heel strike so that no net work is exerted on the c.o.m. The energy which needs to be injected can be determined by analyzing the change in kinetic coenergy which results from the redirection of the velocity of the c.o.m. due to the push off and heel strike.

In the first situation the robot has no feet and energy injection takes place after impact so the required energy to be injected becomes as in equation 1 .

$$
\begin{aligned}
W^{+} & =\frac{1}{2} M\left(v_{\text {com }}^{-}\right)^{2}-\frac{1}{2} M\left(v_{\text {com }}^{+}\right)^{2} \\
& =\frac{1}{2} M\left(v_{\text {com }}^{-}\right)^{2}\left(1-\cos ^{2}(2 \alpha)\right)
\end{aligned}
$$

In the second and third situation the required energy is injected just prior to heel strikeand the required energy injection is given in equation 2. It is clearly visible that the required energy is less when the push off is generated with feet as $\gamma<\alpha$. The physical explanation for this is that due to the curved feet the rotation point prior to impact is shifted forward with respect to the ankle so that the redirection of the velocity of the c.o.m. at heel strike is smaller.

$$
\begin{aligned}
W^{+} & =\frac{1}{2} M\left(v_{\text {com }}^{c}\right)^{2}-\frac{1}{2} M\left(v_{\text {com }}^{-}\right)^{2} \\
& =\frac{1}{2} M\left(v_{c o m}^{-}\right)^{2}\left(\frac{1}{\cos ^{2}(\alpha)}-1\right) \text { (No feet) } \\
& =\frac{1}{2} M\left(v_{c o m}^{-}\right)^{2}\left(\frac{1}{\cos ^{2}(\gamma)}-1\right) \text { (Curved feet) }
\end{aligned}
$$

The timing of the push off is very important. Pushing off too late results in a greater energy loss, as the conditions under which the collision takes place are not optimally

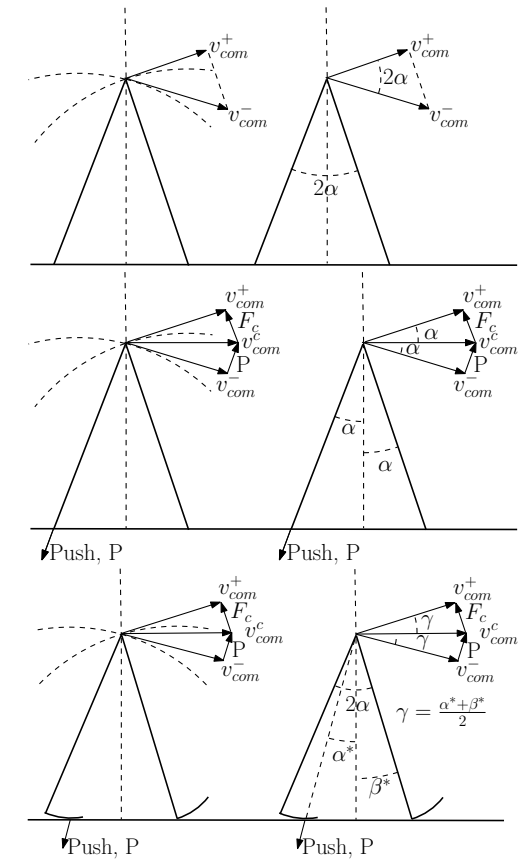

Fig. 1. Effects of a pre-impact push off on the step to step transition where: $v_{\text {com }}^{-}$the velocity of the c.o.m. before heel strike and push off, $v_{\text {com }}^{c}$ the redirected velocity of the c.o.m. due to the pre-impact push off before heel strike, $F_{c}$ redirection of the velocity of the c.o.m. due to heel strike and $v_{\text {com }}^{+}$the velocity of the c.o.m. after heel strike

influenced. However if the push off occurs too early the energy loss is also increased as the downward velocity of the swing leg will start to increase again under the influence of gravity.

\section{Mass distribution}

An important determinant of the robustness of the limit cycle is the ground clearance during the swing phase. With very little ground clearance the robot will already stumble and fall due to small disturbances or objects on the ground. The most effective way to achieve ground clearance is to bend the knee during the swing phase. Bending of the knee is obtained when the rotational velocity of the upper limb around the hip exceeds the rotational velocity of the lower limb with respect to the hip joint.

When the swing phase is fully passive the swing response of the leg is completely determined by the dynamics of the leg under the influence of gravity and the initial state. The dynamic behaviour in turn depends on the configuration of masses in the system.

To determine the optimal mass distribution a robot leg, with lengths of the upper and lower limb of $0.47 \mathrm{~m}$ and $0.4 m$, was simulated as a double pendulum (the ankle is kept rigid during the swing phase). The hip was given an initial angle $(0.5 \mathrm{rad})$ and velocity $(-1 \mathrm{rad} / \mathrm{s})$, which is assumed to correspond with a normal human walking speed [16]. The mass of the upper limb was fixed at $5 \mathrm{~kg}$ and a parameter sweep was performed on the mass of the lower limb and 
foot. The minimum ground clearance for each parameter combination is shown in figure 2 .

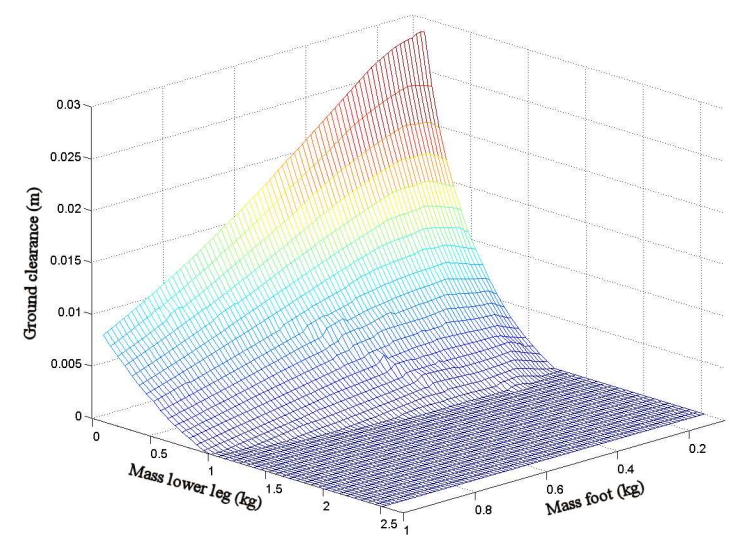

Fig. 2. Ground clearance as function of mass distribution

Figure 2 illustrates that when the mass ratio between upper and lower leg is low there will be no ground clearance and that maximum ground clearance is achieved for the highest ratio. From a construction point of view a good mass ratio is assumed to be 10:1 at which the mass of the foot is chosen to be $0.4 \mathrm{~kg}$ and results in a ground clearance of somewhat more than $1 \mathrm{~cm}$. This mass distribution is assumed to correspond with a robot that obtains sufficient ground clearance during the swing phase and is still physical realizable. The obtained clearance is about the same as the ground clearance in human gaits [2]. It should be noted that the absolute ground clearance depends on the initial velocity of the hip joint. This means that for a lower mass ratio sufficient ground clearance might still be achieved at higher walking speeds. A stable limit cycle can therefore be generated, but over a smaller range of walking speeds.

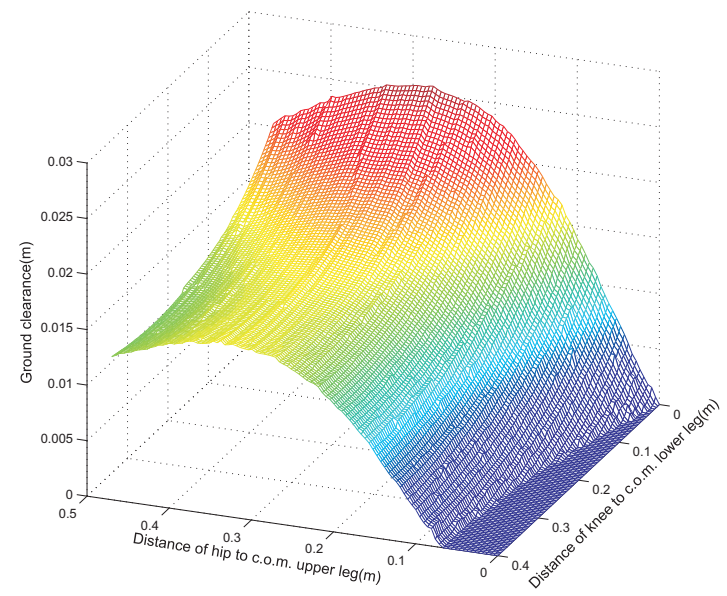

Fig. 3. Ground clearance as function of location of limb c.o.m.

A second parameter sweep has been performed with the chosen mass ratio, but with variable locations of the c.o.m. within the upper and lower leg. In a physical design the location of the c.o.m. of the foot can be influenced the least because the construction has to be strong and the foot is in comparison smallest in size, so its location is fixed in this analysis. Figure 3 shows the dependency of the ground clearance on the locations of the c.o.m. in the upper and lower limb. Figure 3 shows that ground clearance is maximized when the c.o.m. of the lower leg is located very close to the knee. For maximum knee flexion the angular acceleration of the knee joint should be maximized. This is the case when the moment of inertia of the lower limb around the knee is minimized, i.e. minimizing the distance of the c.o.m. of the lower leg to the knee.

Two opposing effects determine the optimal location of the c.o.m. of the upper limb. On the one hand the kinetic energy the system contains at the beginning of the swing phase given the initial conditions is maximized when the c.o.m. of the upper limb is located near the knee. On the other hand angular acceleration around the hip due to gravity is maximized when the c.o.m. is located near the hip (smallest moment of inertia). Figure 3 shows that these effects are approximately equally beneficial and that therefore the optimal location of the c.o.m. is in the middle of the limb.

\section{Foot shape}

The foot is of great importance during walking. It carries the weight of the body forward during the stance phase and it acts as a rigid lever during push off. During the stance phase the human foot undergoes an elastic deformation while it carries the body weight forward. This elastic deformation results in a particular roll over shape. Intersubject biomechanical studies have shown that this roll over shape has a low variance and has a curvature of $30-35 \mathrm{~cm}$ [9]. This result was obtained by applying a coordinate transformation on the center of pressure (c.o.p.) during walking from the world frame to the ankle frame. This is sketched in figure 4 where the center of the roll over shape (c.o.r.o.s) is also drawn.

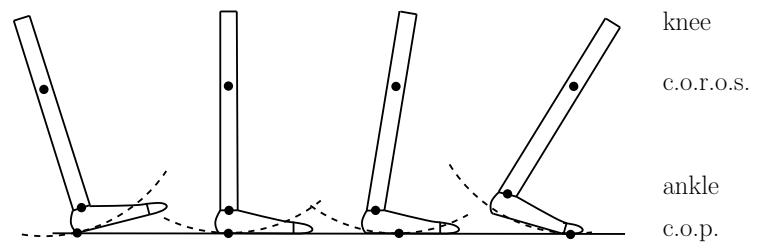

Fig. 4. Human roll over shape of the foot

In [14] computer models were used to analyze how the foot curvature influences the local stability of passive dynamic walkers. The optimal curvature with respect to energy efficiency was found to be $\frac{1}{3}$ of the leg length, which corresponds approximately to the human roll over shape. In [1] a model of a powered passive dynamic walker with knees and curved feet was analyzed. They used this model to 
deterimine the influence of the curvature of the foot on the mechanical work needed per step. They concluded that for the model with knees and curved feet the optimal curvature with respect to energy efficiency was $38 \%$ of the leg length.

In physical robots the lowest point of the curvature of the roll over shape (the nadir) is often shifted forward with respect to the ankle. This offset is not present in the human roll-over shape. In [14] it was concluded that with this offset the passive reaction torque helps to keep the knee locked during the stance phase. Powered dynamic walkers have an active locking mechanism in the knee and therefore would not need this offset in the nadir to keep the knee locked. In [17] and [4] an offset in the nadir is however found essential to stabilize the limit cycle of those robots.

As was discussed earlier the upper limb should be much heavier than the lower limb to avoid foot scuffing during the swing phase. At approximately 50\% of the swing phase the upper limb of the swing leg passes the stance leg. As the upper limb is much heavier than the lower limb this causes the c.o.m. of the entire robot to shift forward to a position in front of the ankle. Robots equipped with circular feet without an offset in the nadir would immediatly start to rotate forward due to the gravitional force acting on the c.o.m., figure 5. This results in an acceleration of the c.o.m. in the direction of movement (forward acceleration) and causes the effective step length to be decreased (the angle of the hip will be smaller at the end of the step than at the beginning). This is a vicious cycle and will eventually cause the robot to trip and fall.

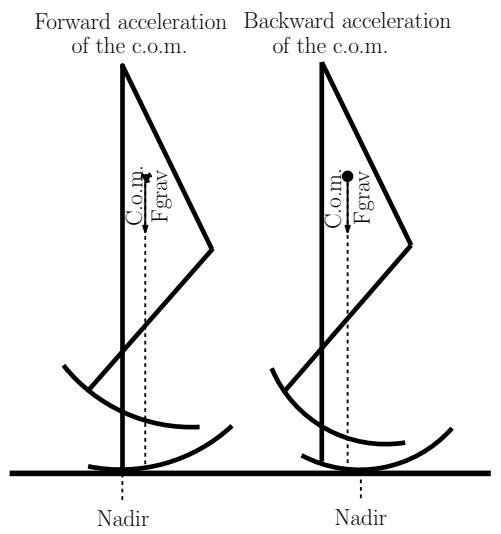

Fig. 5. Rolling of stance leg due to location of c.o.m.

With an offset of the nadir the c.o.m. is kept behind the nadir longer, figure 5. When the c.o.m. is behind the nadir the gravitional force on the c.o.m. works to decelerate the stance leg. This effectively decreases the velocity of the c.o.m. in the direction of movement (backward acceleration) and provides the extra time needed for the swing leg to finish the complete swing phase so that the conditions at the end of the swing phase are symmetric to those at the beginning.

\section{E. Human dynamics}

Ankle actuation is very important in the human gait. Studies have shown that $80 \%-85 \%$ of the mechanical energy generated in the gait cycle is generated in the ankle during the push off of the stance leg [16]. There exist however large differences between the dynamics of the human body and the results presented in this section.

The most prominent difference is the mass distribution in the humans legs. In section II-C it was shown that sufficient ground clearance is achieved with a mass ratio of 10:1 between the upper and lower limb. The mass ratio between the human upper and lower limb however is only 2:1 [15] with which on basis of figure 2 no ground clearance would be achieved. Several reasons why humans can walk mostly powered by ankle actuation and a low mass ratio are:

1) The presence of joint spanning muscles Push off in the human gait is obtained by contraction of the soleus and the gastrocnemius muscles (the deep and superficial calf muscles)[11]. Contraction of the gastrocnemius muscle however will also result in bending of the knee as it is attached to the femur. Since there is an initial bending of the knee at the beginning of the swing phase less bending needs to be obtained during the swing phase to reach a desired knee flexion when the swing leg passes the stance leg. Therefore a lower mass ratio suffices.

2) The swing phase is not completely passive Humans use the muscles in their thighs to thrust the swing leg forward [16]. The acceleration of the upper leg contributes to the bending of the knee.

Another difference, as can be seen in figure 4 , is that the offset of the nadir is not present in the human roll-over shape. Three contributing factors that this offset is not needed in the human roll-over shape are:

1) The elastic deformation of the foot during the stance phase

Kinetic energy is dissipated and partially stored in the elastic deformation of the foot which results in a decrease of the velocity of the c.o.m. of the person.

2) The presence of an upper body

One leg constitutes just over $20 \%$ of the human body mass [15]. The passing of the upper part of the swing leg will therefore result in a smaller forward displacement of the c.o.m.

3) The swing phase is not completely passive Due to the forward acceleration of the swing leg a negative reaction torque is applied on the stance leg. This effictively slows down the stance leg.

In human gait the push off also starts before heel strike and continues through a double support. The human push off is likely triggered by pressure loading of the fore foot and/or pressure build up in the achilles tendon, which both occur after heel rise.

\section{SIMULATION MODEL}

To construct and simulate dynamic models the simulation software 20-sim is used [3]. The internal 3D Mechanics Editor can be used to generate code for complex linkages of rigid bodies. 


\section{A. Robot model}

A model of a 5 d.o.f. bipedal robot was constructed. The model consists of 6 rigid bodies, representing the legs and feet, the hip is assumed massless. The c.o.m. of the upper and lower leg is located in the middle of each link and the c.o.m. of the foot has a forward displacement with respect to the ankle, figure 6 . The dimensions of the robot are presented in table I and are chosen to correspond with a robot of humanlike dimensions. The radius of the foot is chosen at $12 \%$ of the leg length, which is smaller than the theoretical optimal $\frac{1}{3}$ of the leg length from [14]. The offset of the nadir of the roll over shape flattens the foot in longitudinal direction, which decreases ground clearance during the swing phase especially at lower walking speeds. A stronger curvature of the foot was found to prevent scuffing of the foot during the swing phase.

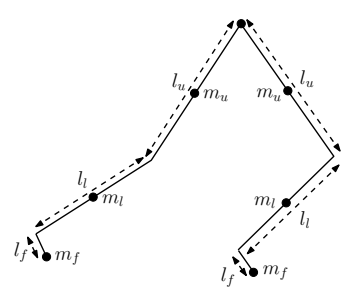

(a) Configuration model

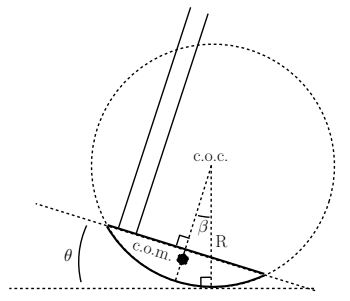

(b) Contact point calculation
Fig. 6. Robot model

\begin{tabular}{|c|c|c|c|c|}
\hline & \multicolumn{2}{|l|}{ Length (m) } & \multicolumn{2}{|c|}{ Mass (kg) } \\
\hline Upper leg & \multicolumn{2}{|l|}{$0.47\left(l_{u}\right)$} & \multicolumn{2}{|c|}{$5\left(m_{u}\right)$} \\
\hline Lower leg & \multicolumn{2}{|l|}{$0.4\left(l_{l}\right)$} & \multicolumn{2}{|c|}{$0.5\left(m_{l}\right)$} \\
\hline & Radius (m) & $\begin{array}{l}\text { Offset nadir } \\
(\mathrm{m})\end{array}$ & $\begin{array}{l}\text { Height } \\
\text { (m) }\end{array}$ & Mass (kg) \\
\hline Foot & $0.106(R)$ & $0.05\left(l_{f}\right)$ & 0.05 & $0.4\left(m_{f}\right)$ \\
\hline
\end{tabular}

TABLE I

DIMENSIONS OF ROBOT MODEL

\section{B. Contact model}

The impact of the foot with the floor is modelled as a critically damped elastic collision. The Hunt-Crossley contact model [10] is used to calculate the normal force which is exerted on the foot by the ground. The Hunt-Crossley model is given in equation 3 where $\mathrm{K}$ is the spring constant $\mathrm{D}$ the dampening factor, $\mathrm{z}$ the lowest point of the foot, $\dot{z}$ the vertical velocity of the lowest point of the foot and $H_{f}$ the height of the ground.

$$
F_{(N)}= \begin{cases}-K\left(z-H_{f}\right)+\left(z-H_{f}\right) D \dot{z} & \text { if } z \leq H_{f} \\ 0 & \text { if } z>H_{f}\end{cases}
$$

Single point contact at the position on the foot which is closed to the ground is assumed. In [7] it was shown how for several foot shapes the lowest point with respect to the ground can be calculated. The used geometry is shown in figure 6 in which $\mathrm{R}$ is the radius of the roll over shape and c.o.c. the center of the circle spanned by the roll over shape.

\section{Controller}

One of the characteristics of robots based on passive dynamics is that they can produce a stable gait with very simple controllers. The push off is generated by a P-controller which drives the ankle towards a setpoint. The ankle setpoint and controller gain are used to tune the energy which is injected into the system during each push off phase. During the simulations the ankle setpoint had a range of $[0.15,0.3] \mathrm{rad}$ and the controller gain had a range of $[55,80] \mathrm{N} / \mathrm{rad}$.

When the setpoint has been reached a PD-controller is used to retract the ankle towards the stationary position. The differential action damps out possible oscillations of the foot around the setpoint. A vibrating foot during the swing phase is undesirable because it can decrease ground clearance.

\section{SIMULATION RESULTS}

To validate the concept before physical construction, simulations have been performed with two different instants at which the push off is initiated. In the first type of simulations the push off was iniated after heel strike. This moment can be determined very precisely in real life and is therefore often applied in physical systems [4][6][17]. In section II it was discussed that less mechanical work is required when the actuation takes place before impact. Simulations have been carried out in which the actuation is initiated when the foot of the swing leg was $1 \mathrm{~cm}$ above the floor.

Powered passive dynamic walkers are usually compared on the specific energetic and specific mechanical cost of transport $\left(C_{e t}\right.$ and $\left.C_{m t}\right)$. The $C_{e t}$ uses the total energy required for walking and $C_{m t}$ uses the mechanical work which omits the negative influence of the energy loss in the physical transmission. As in this model no physical transmission is modelled the $C_{e t}$ equals the $C_{m t}$. The $C_{m t}$ is calculated as in equation 4 , in which $P_{m}$ is the mechanical power, $g$ the gravitational constant, $m$ the mass of the robot and $v$ the forward velocity of the robot.

$$
\begin{aligned}
P_{m} & =\frac{\int_{t=0}^{t_{0}} T \omega d t}{t_{0}} \\
C_{m t} & =\frac{P_{m}}{g m v}
\end{aligned}
$$

The $C_{m t}$ has been determined for a wide range of velocities at which the two models produced a stable gait and are given in figure 7 . The $C_{m t}$ ranges from 0.03 to 0.08 , which makes the model very energy efficient [5].

These simulation results are approximately verified by experimental results discussed in [4]. An ankle actuated robot of similar configuration as the model used in this paper is discussed which weighs $12.7 \mathrm{~kg}$ and walks at $0.44 \mathrm{~m} / \mathrm{s}$ with 3 Watts of mechanical power. If the absence of friction is taken into account this agrees with the results presented in figure 7.

Figure 7 clearly shows that initiating the push-off before impact results in a more energy efficient gait. The required energy is about $75 \%$ of that with post-impact actuation. This however is still much higher than the predicted $25 \%$ 


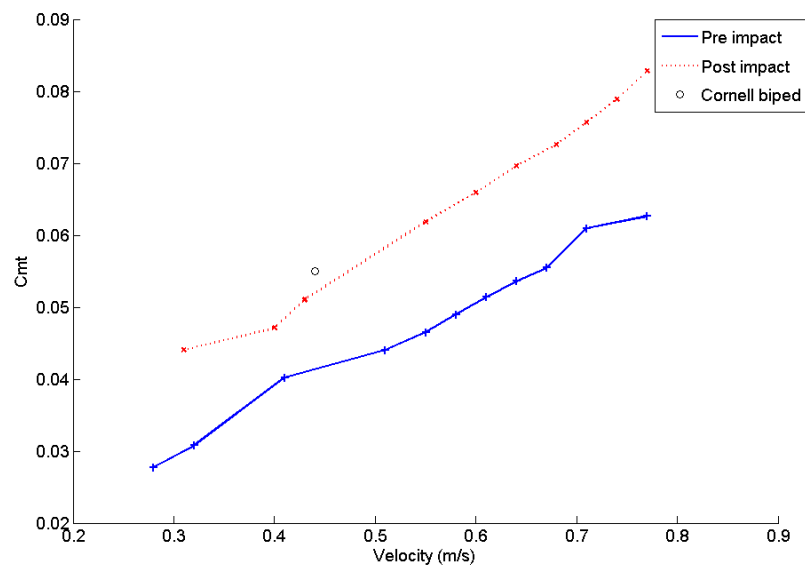

Fig. 7. Specific mechanical cost of transport as function of velocity

by [13] and [12]. The moment at which the actuation is initiated here has not been optimized, the push off force favorably influences the collision conditions but most of the energy injection occurs after heelstrike. Therefore it is assumed that more energy reduction can be achieved. As the mechanical power is not delivered instantaneous in this model, the maximum attainable energy reduction is probably less than discussed in [13] and [12].

The gait produced by especially the pre-impact push off model is very natural looking. In figure 8 a single step of the left leg is broken down into 5 composures. The model exhibits period-1 gaits, so both steps are symmetrical.

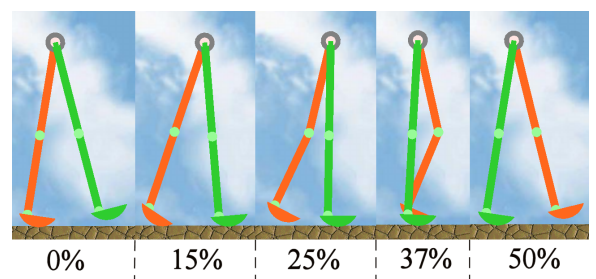

Fig. 8. Composure of robot at percentages of stride time

\section{CONCLUSIONS}

In this paper the design characteristics of robots based on passive dynamic walkers only actuated by ankle actuation were discussed. The mass distribution and foot shape are important factors for the stability and robustness of the limit cycle whereas the moment of push off can lead to a reduction of the required energy to sustain the limit cycle. Simulation results have been presented which indicate that robots complying with these design characteristics can walk over a wide range of velocities at low mechanical cost of transport. The used method of pre-impact push off led to a simulated decrease in required mechanical energy of $25 \%$.

\section{FUTURE WORK}

Based on the results discussed in this paper an ankle actuation system has been designed for the bipedal walker at the Control Engineering group of the University of Twente, figure 9 [8]. It will be used to validate the results discussed in this paper. The walker will also be used as a test bed for new efficient strategies of pre-impact push-off and hybrid combinations of ankle and hip actuation schemes which will result from future research.

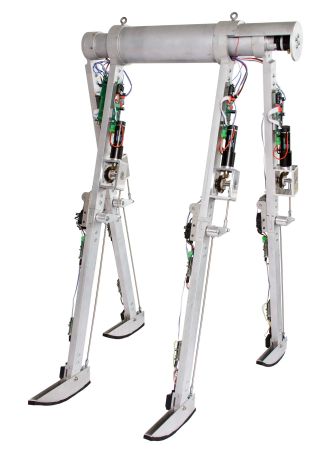

Fig. 9. Dribbel with added feet and ankle actuation system (Photo taken by M.H. Schwirtz)

\section{REFERENCES}

[1] P. G. Adamczyk, S. H. Collins, and A. D. Kuo, "The advantages of a rolling foot in human walking," The Journal of Experimental Biology, vol. 209, pp. 3953-3963, 2006.

[2] E. Ayyappa, "Normal human locomotion, part 2: motion, ground reaction force and muscle activity," American Academy of Orthotists and Prosthetists, vol. 9, no. 2, pp. 42-57, 1997.

[3] C. P. B.V., "20-sim version 3.6," http://www.20sim.com/, 2007.

[4] S. Collins and A. Ruina, "A bipedal walking robot with efficient and human-like gait," IEEE International Conference on Robotics and Automation, pp. 1983-1988, 2005.

[5] S. Collins, A. Ruina, R. Tedrake, and M. Wisse, "Efficient bipedal robots based on passive-dynamic walkers," Science, vol. 307, no. 5712, pp. 1082-1085, February 2005.

[6] E. Dertien, "Dynamic walking with dribbel," IEEE robotics and automation magazine, vol. 13(3), pp. 118-121, 2006.

[7] V. Duindam, "Port-based modelling and control for efficient bipedal walking robots," Ph.D. dissertation, University of Twente, The Netherlands, 2006, iSBN 90-365-2318-4.

[8] M. Franken, "Ankle actuation for planar bipedal robots," Master's thesis, University of Twente, The Netherlands, April 2007.

[9] A. H. Hansen, "Roll-over characteristics of human walking with applications for artificial limbs," Ph.D. dissertation, Northwestern University, Evanston, 2002.

[10] K. Hunt and F. Crossley, "Coefficients of restitution interpreted as damping in vibroimpact," Applied Mechanics, pp. 440-445, 1975.

[11] V. T. Inman, H. J. Ralston, and F. Todd, Human Walking. Williams \& Wilkins, 1981.

[12] A. D. Kuo, "Energetics of actively powered locomotion using the simplest walking model," Journal of Biomechanical Engineering, vol. 124, pp. 113-120, February 2002.

[13] A. D. Kuo, J. M. Donelan, and A. Ruina, "Energetic consequences of walking like an inverted pendulum: step-to-step transitions," Exercise and sport sciences reviews, vol. 33, no. 2, pp. 88-97, April 2005.

[14] T. McGeer, "Passive dynamic walking," International Journal of Robotics Research, vol. 9, no. 2, pp. 62-82, 1990.

[15] S. Pheasant, Body Space: Anthropomorphy, Ergonomics and Design. Taylor \& Francis, 1986.

[16] D. A. Winter, The Biomechanics and motor control of human gait. University of Waterloo Press, 1987.

[17] M. Wisse and J. van Frankenhuyzen, "Design and construction of mike: a 2 d autonomous biped based on passive dynamic walking," International Conference on Adaptive Motion of Animals and Machines, 2003. 